Bulletin of Mathematical Biology (2002) 64, 1083-1099

doi:10.1006/bulm.2002.0313

Available online at http://www.idealibrary.com on IDE $\mathbf{A}^{[}$

\title{
A Mathematical Model of Human Atrioventricular Nodal Function Incorporating Concealed Conduction
}

\author{
PETER JØRGENSEN AND CARSTEN SCHÄFER \\ Center for Nonlinear Dynamics, Department of Physiology, \\ McGill University, \\ 3655 Drummond St., \\ Montréal, Québec, \\ Canada H3G1Y6 \\ PETER G. GUERRA, MARIO TALAJIC AND STANLEY NATTEL \\ Montréal Heart Institute, \\ 5000 Bélanger St., \\ Montréal, Québec, \\ Canada H1T 1C8
}

\section{LEON GLASS*}

Center for Nonlinear Dynamics, Department of Physiology,

McGill University,

3655 Drummond St.,

Montréal, Québec,

Canada H3G1Y6

E-mail: glass@cnd.mcgill.ca

This work develops a mathematical model for the atrioventricular (AV) node in the human heart, based on recordings of electrical activity in the atria (the upper chambers of the heart) and the ventricles (the lower chambers of the heart). Intracardiac recordings of the atrial and ventricular activities were recorded from one patient with atrial flutter and one with atrial fibrillation. During these arrhythmias, not all beats in the atria are conducted to the ventricles. Some are blocked (concealed). However, the blocked beats can affect the properties of the AV node. The activation times of the atrial events were regarded as inputs to a mathematical model of conduction in the AV node, including a representation of $\mathrm{AV}$ nodal concealment. The model output was compared to the recorded ventricular response to search for and identify the best possible parameter combinations of the model. Good agreement between the distribution of interbeat intervals in the model and data for durations of $5 \mathrm{~min}$ was achieved. A model of $\mathrm{AV}$ nodal behavior during atrial flutter and atrial fibrillation could potentially help to understand the relative roles of atrial input activity and intrinsic $\mathrm{AV}$ nodal properties in determining the ventricular response.

(C) 2002 Society for Mathematical Biology. Published by Elsevier Science Ltd. All rights reserved.

*Author to whom correspondence should be addressed.

0092-8240/02/061083 + $17 \quad \$ 35.00 / 0$

Elsevier Science Ltd. All rights reserved.

(C) 2002 Society for Mathematical Biology. Published by 


\section{INTRODUCTION}

Atrial flutter and atrial fibrillation are abnormal cardiac rhythms in which there are unusually rapid rhythms in the atria, the upper chambers of the heart. In atrial flutter, the rate is fast and regular whereas in atrial fibrillation the rate is fast, but irregular. The rapid activity in the atria is filtered by the atrioventricular (AV) node, which is the only conducting pathway between the atria and the ventricles (the lower chambers of the heart) in the normal heart. Consequently, during atrial flutter and atrial fibrillation only a fraction of the atrial activations is transmitted to the ventricles. Atrial activations that fail to propagate to the ventricles nevertheless play a crucial role in determining the ventricular response. These atrial activations partially penetrate the AV node and thus affect the electrophysiological state of AV node (Langendorf, 1948; Moe and Abildskov, 1964; Pick and Langendorf, 1979). The effects of these concealed impulses on the electrophysiological properties of the AV node are still not completely understood.

Lewis and Master (1925) showed that the conduction time of a single premature stimulus through the AV node decreased as the time interval since the last passage of a previous impulse through the AV node increased. The function describing this relationship, the AV nodal recovery curve, can be used to predict the rhythms that arise during atrial stimulation at a rate faster than the intrinsic rate (Decherd and Ruskin, 1946; Shrier et al., 1987). Further work has developed models of $\mathrm{AV}$ nodal function describing the response to both periodic and irregular inputs (Heethaar et al., 1973a,b; Cohen et al., 1983; Talajic et al., 1991; Meijler et al., 1996; Zeng and Glass, 1996). In this work however, there is no agreement on how blocked beats affect subsequent conduction through the AV node. Furthermore, it is impossible to measure the $\mathrm{AV}$ nodal recovery curve during sustained atrial flutter and atrial fibrillation.

The current work develops a theoretical approach to model AV nodal function by comparing the observed ventricular response during atrial flutter and fibrillation to the predictions of the model. Such an analysis could be applied to better understand the ventricular response to atrial flutter and atrial fibrillation in man.

\section{METHODS}

Recording and preprocessing of data. Recordings were carried out in two patients referred for catheter ablation of chronic atrial arrhythmias. One patient was in chronic atrial flutter, whereas the second patient was in chronic atrial fibrillation. Two quadripolar electrode catheters $(1 \mathrm{~cm}$ interelectrode spacing) were used: one in the right ventricular apex, and a second in the right atrium within Koch's triangle. Electrograms were amplified and filtered (high pass $30 \mathrm{~Hz}$, low pass $250 \mathrm{~Hz}$ ), were digitized at $1 \mathrm{KHz}$, and were recorded onto a disk. The data files were analyzed in segments of $5 \mathrm{~min}$ intervals (Task Force, 1993). Custom-written software 

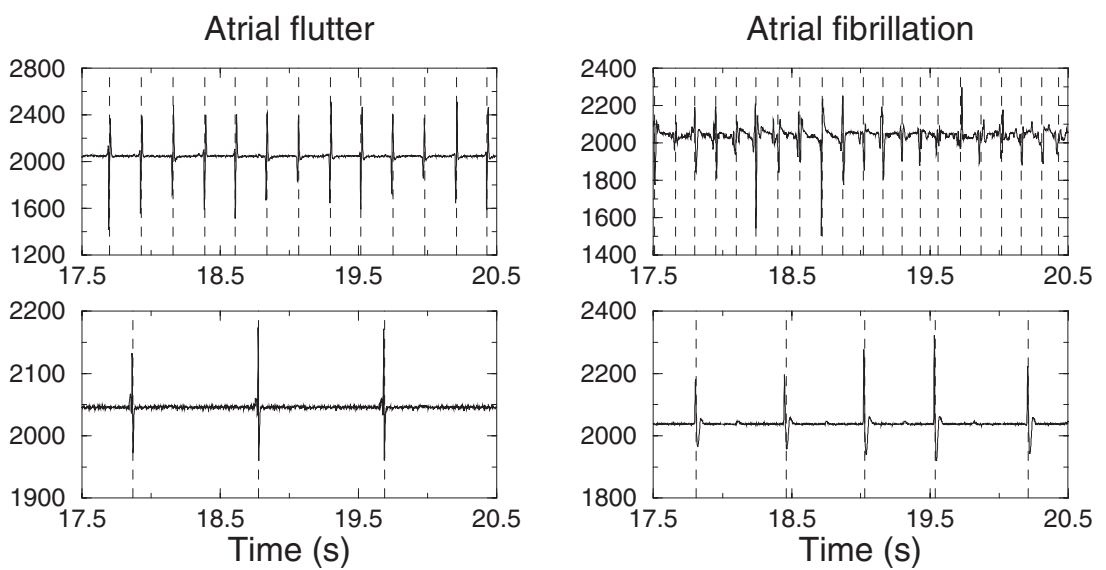

Figure 1. The extracted activation times (dashed lines) from the preprocessing are superposed onto the recordings (solid lines) in order to graphically verify the extraction method. The top row shows the atrial signals, and the bottom row the ventricular signals. The left and right columns show the atrial flutter and atrial fibrillation sets, respectively.

was developed for the purpose of extracting the activation times of the atrial and ventricular activation complexes. The following sequence of steps was taken to determine the atrial activation times during atrial fibrillation. The derivative was computed and smoothed by the Savitzky-Golay filter (Press et al., 1993) which is a low-pass filter well-suited to preserve heights and widths of narrow features such as the events in intracardiac recordings. Depolarizing and repolarizing parts of the activation complexes are integrated into large complexes by first taking the absolute value of the resulting time series and then performing a moving average window. These complexes are fitted to second degree polynomials by using the Savitzky-Golay filter of second degree. The smallest maxima of the resulting time series are eliminated by using a moving average. The activation times are finally identified as the remaining maxima of the time series. The result of the described preprocessing method is verified manually (Fig. 1). Preprocessing the intraatrial recording of atrial flutter and the intraventricular recordings is simpler due to the more distinct activation complexes of these recordings compared to the atrial signal of atrial fibrillation.

\section{Mathematical model of concealed conduction.}

Models of $A V$ nodal function. We develop a theoretical model to predict an output sequence of ventricular activations on a beat-to-beat basis given an input sequence of extracted atrial activation times from either the atrial flutter or atrial fibrillation recordings. We propose an iterative model which uses two fundamental properties of the AV node to predict the ventricular response. After the conduction of an atrial impulse through the AV node to the ventricles, the AV node has a refractory period $\theta$. If an atrial activation reaches the AV node after the end of 


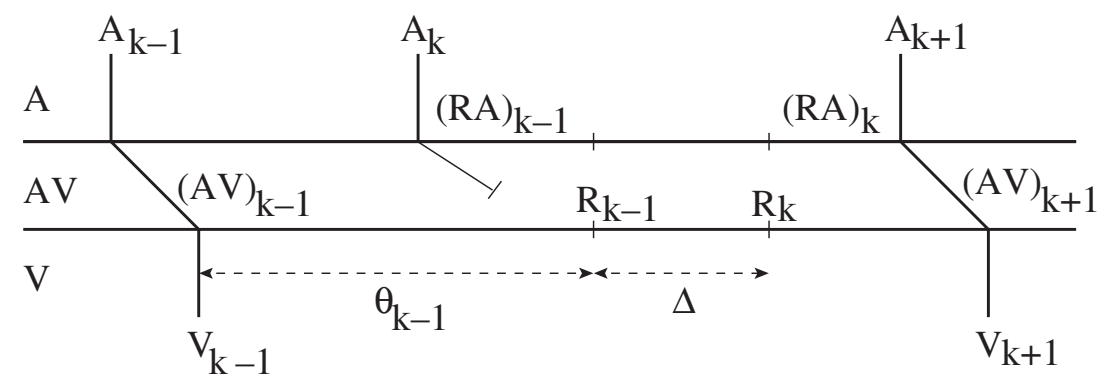

Figure 2. Schematic illustration of the effect of a blocked event on AV nodal conduction. The event $A_{k}$ reaches the $\mathrm{AV}$ node during the refractory period owing to a conducted atrial event $A_{k-1}$. As a consequence the refractory period $\theta_{k-1}$ is prolonged a time interval $\Delta$. The new refractory period is thus $\theta_{k}=\theta_{k-1}+\Delta$. The next atrial event $A_{k+1}$ arrrives after the expiration of the new refractory period, and it is thus conducted through the AV node to the ventricles with a conduction time $(A V)_{k+1}$. Notice that the recovery time $(R A)_{k}$ used as the independent variable in the computation of $(A V)_{k+1}$ is computed from the end of the refractory period $R_{k}$ to $A_{k+1}$.

the refractory period, that activation is conducted through the AV node to give a ventricular activation. Activations that arrive at the AV node during the refractory period are blocked (concealed) and lead to an increment in the refractory period by an amount $\Delta$. Figure 2 shows a schematic illustration of the model.

Our notation follows. We start with a conducted beat, $A_{k-1}$ in Fig. 2, that is associated with a ventricular activation $V_{k-1}$. The associated $\mathrm{AV}$ conduction time is designated by $(A V)_{k-1}$. Following the ventricular activation, the AV node will be refractory for a time interval $\theta_{k-1}=\theta$. If the next atrial activation $A_{k}$ falls during the refractory period, then

$$
\theta_{k}=\theta_{k-1}+\Delta
$$

The refractory period is always measured from the time of the last conducted ventricular impulse which in this case is $V_{k-1}$. We assume that the conduction time through the AV node depends on the recovery time from the end of the preceding refractory period. For example, in Fig. 2, the conduction time of the $(k+1)$ st atrial activation is given by

$$
A V_{k+1}=f\left((R A)_{k}\right)=A V_{\min }+\alpha \exp \left(-(R A)_{k} / \tau\right)
$$

where the parameter $A V_{\min }$ is the minimal conduction time through the $\mathrm{AV}$ node, and $\alpha$ and $\tau$ are positive constants (Shrier et al., 1987; Talajic et al., 1991). Thus, starting with the input sequence of atrial activations and an initial ventricular activation, an output sequence of ventricular activations can be generated iteratively once parameter values are set. 
Model assessment. To assess the mathematical model, a sequence of computed ventricular activations $V_{k}^{\text {model }}$ is compared to the recorded sequence $V_{l}^{\text {rec }}$ in which the subscripts $k, l$ index the sequences. It is convenient to transform the two event series into time series of interbeat intervals, $V V_{k-1}^{\text {model }}$ and $V V_{l-1}^{\text {rec }}$, and to make comparisons based on those. We need to compare two nonequidistantly sampled, irregular time series with each other. We have implemented two methods of assessing the model agreement.

One method is to compute the average distance between the discrete events series (DES) of the two time series $V V_{k-1}^{\text {model }}$ and $V V_{l-1}^{\text {rec }}$. The two time series $V V_{k-1}^{\text {model }}$ and $V V_{l-1}^{\text {rec }}$ are at first resampled at the same equidistantly spaced times. We resample at times $t_{i}$, where $d t=t_{i}-t_{i-1}$ is $1 / 10$ of the length of the smallest interbeat interval in either of the two time series. An integration along the time axis is then performed in order to calculate the average distance $D_{T}$ between the two linearly interpolated time series. We call $D_{T}$ the area measure, and with this measure local disturbances, e.g., extra beats $(k \neq l)$ in one time series have only local consequences in the computation.

Another method is to apply the Kolmogorov-Smirnov test (Press et al., 1993) to assess the similarity of the distributions of $V_{k}^{\text {model }}$ and $V_{l}^{\text {rec }}$. The Kolmogorov-Smirnov test tests against the null hypothesis that the population distribution functions of the time series $V V_{k}^{\text {model }}$ and $V V_{l}^{\text {rec }}$ are the same. The result of the Kolmogorov-Smirnov test $p$ is the significance level for the null hypothesis. A small $p$ value means that the cumulative distribution function of $V V_{k}^{\text {model }}$ is significantly different from that of $V V_{l}^{\text {rec }}$. A high $p$ value $(p \leq 1)$ means that the null hypothesis cannot be rejected. Hence, a high $p$ value does not mean that the two distribution functions are the same, but rather from a statistical standpoint we cannot say they are different (Press et al., 1993).

\section{RESULTS}

\section{Atrial flutter.}

Data analysis. Key statistical numbers of the atrial flutter data are summarized in Table 1. The average interatrial interval is $A A_{\text {mean }}=229 \pm 5 \mathrm{~ms}$, and the average interventricular interval is $V V_{\text {mean }}=917 \pm 7 \mathrm{~ms}$, thus reflecting a $4: 1$ conduction ratio between atrial and ventricular rhythms. The $4: 1$ conduction ratio persists throughout the recording time. The autocorrelation function of the interatrial intervals shows that the atrial rhythm is periodic with a period of four (Fig. 3). The autocorrelation function of the interventricular intervals indicates the presence of a trend in the data. The interbeat intervals (time duration between two consecutive activations) are plotted against time (Fig. 4) (Task Force, 1993). The atrial rhythm during atrial flutter is not constant, but subject to small beat-tobeat cycle length variations possibly owing to the effects of ventricular contraction (Wells et al., 1979; Waxman et al., 1991; Pavri et al., 1999). 
Table 1. Statistics of atrial flutter data.

\begin{tabular}{lccc}
\hline Intervals & \multicolumn{1}{c}{$A A$} & $V V^{\text {rec }}$ & $V V^{\text {model }}$ \\
\hline Number of intervals & \multicolumn{1}{c}{1308} & \multicolumn{1}{l}{326} & \multicolumn{1}{l}{324} \\
Minimum & $194 \mathrm{~ms}$ & $890 \mathrm{~ms}$ & $901 \mathrm{~ms}$ \\
Maximum & $257 \mathrm{~ms}$ & $956 \mathrm{~ms}$ & $938 \mathrm{~ms}$ \\
Average & $229 \mathrm{~ms}$ & $917 \mathrm{~ms}$ & $917 \mathrm{~ms}$ \\
Standard deviation & $5 \mathrm{~ms}$ & $7 \mathrm{~ms}$ & $6 \mathrm{~ms}$ \\
\hline
\end{tabular}
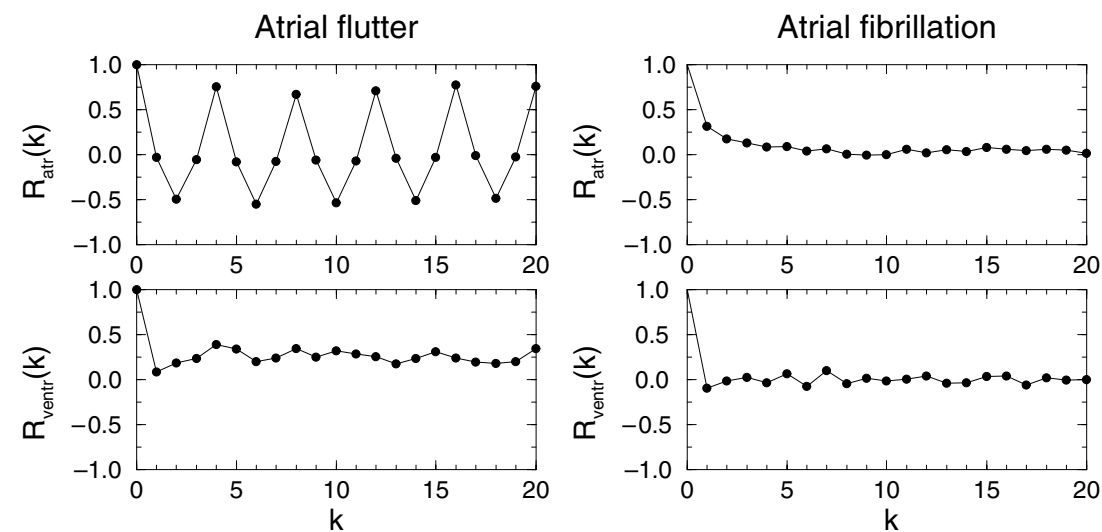

Figure 3. Autocorrelation functions $\mathrm{R}(\mathrm{k})$ as a function of the lag $\mathrm{k}$. The left and right columns show the atrial flutter and atrial fibrillation sets, respectively.
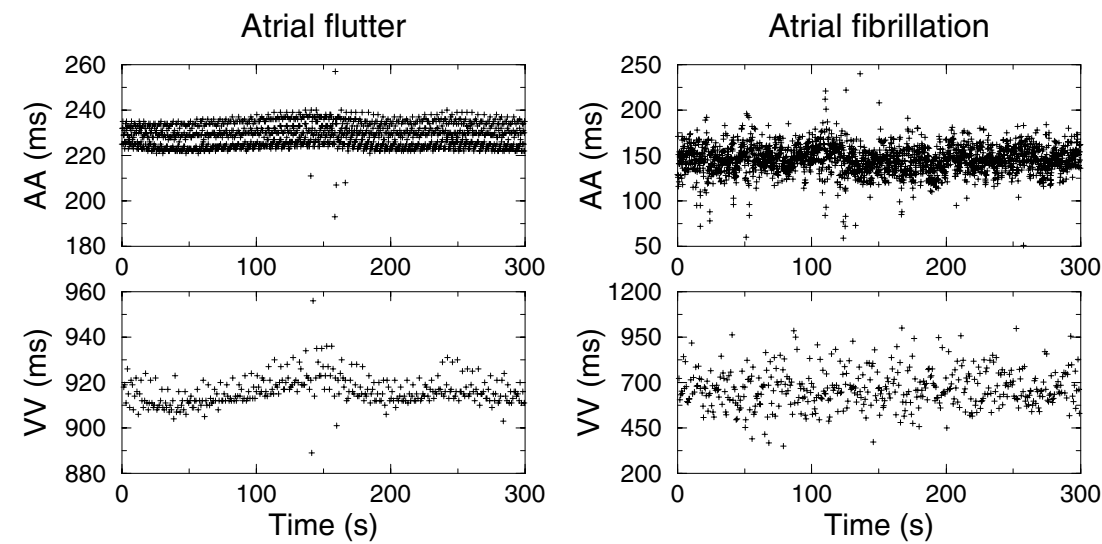

Figure 4. The DES for the atrial and ventricular activities are plotted in the top and bottom rows, respectively. The left column shows the atrial flutter DES, and the right column the atrial fibrillation DES.

A priori, it is not clear which atrial activations are conducted to the ventricles during the atrial flutter. Although there is no definite way to determine based on the current data, the following method offers a statistical approach. We can group the atrial activations into blocks of four consecutive beats in four different 

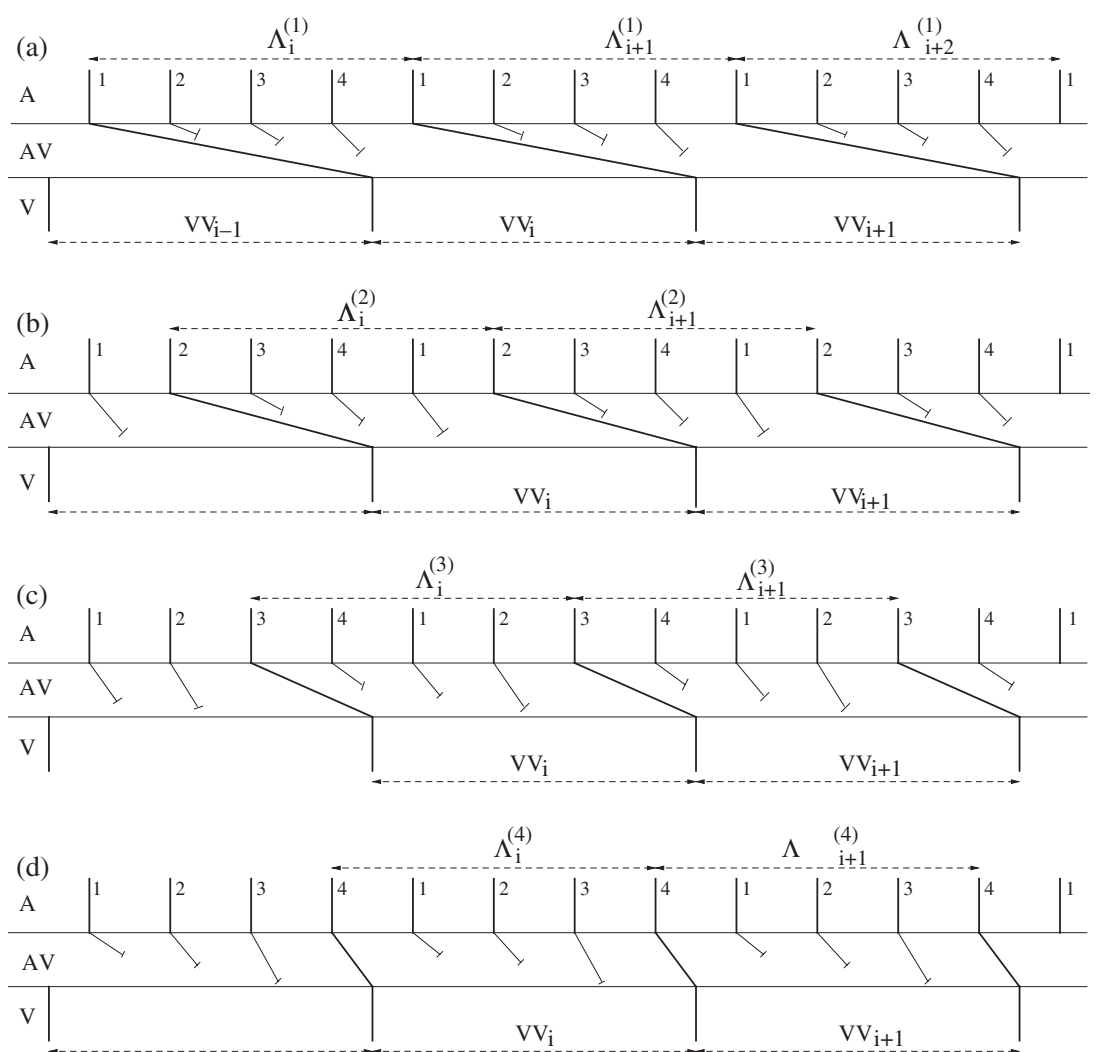

Figure 5. Atrial flutter. The figure consists of four panels, each schematically displaying a ladder diagram of a possible interpretation of the atrial flutter data. Atrial events are pooled into groups of four. Panel (a): 1st activation is conducted through the AV node, and the last three events are blocked. Panels (b)-(d): 2nd-4th activations are conducted, respectively, while remaining events are blocked. The $\Lambda$ 's are the grouped interatrial intervals, and the $V V$ 's are the interventricular intervals.

ways as indicated schematically in Fig. 5. We then obtain the following four sequences of values $\Lambda_{i}^{(1)}, \Lambda_{i}^{(2)}, \Lambda_{i}^{(3)}, \Lambda_{i}^{(4)}$, where the subscript $i$ is associated with a corresponding ventricular interval $V V_{i}$.

For each of the four interpretations of which atrial event is conducted, the interventricular intervals $V V_{i}$ are plotted as a function of the preceding grouped interatrial intervals $\Lambda_{i}$ (Fig. 6). The correlation coefficent between $\Lambda^{(4)}$ and $V V$ is $\rho_{4}=0.85$ with a slope of regression of $\alpha_{4}=0.91$, which is markedly better than for the other three cases. See Fig. 6 for all correlation and regression coefficients. Therefore, interpretation D (Fig. 5) in which the atrial events labeled 4 are conducted to the ventricles is the most likely interpretation of the atrial flutter data. Under the assumption of interpretation $\mathrm{D}$ we can compute the mean conduction time to be $A V_{\text {mean }}=164 \pm 4 \mathrm{~ms}$. During sinus rhythm in the same patient (cycle length of $730 \mathrm{~ms}$ ), the $\mathrm{AV}$ interval was $120 \mathrm{~ms}$, so that the conduction 

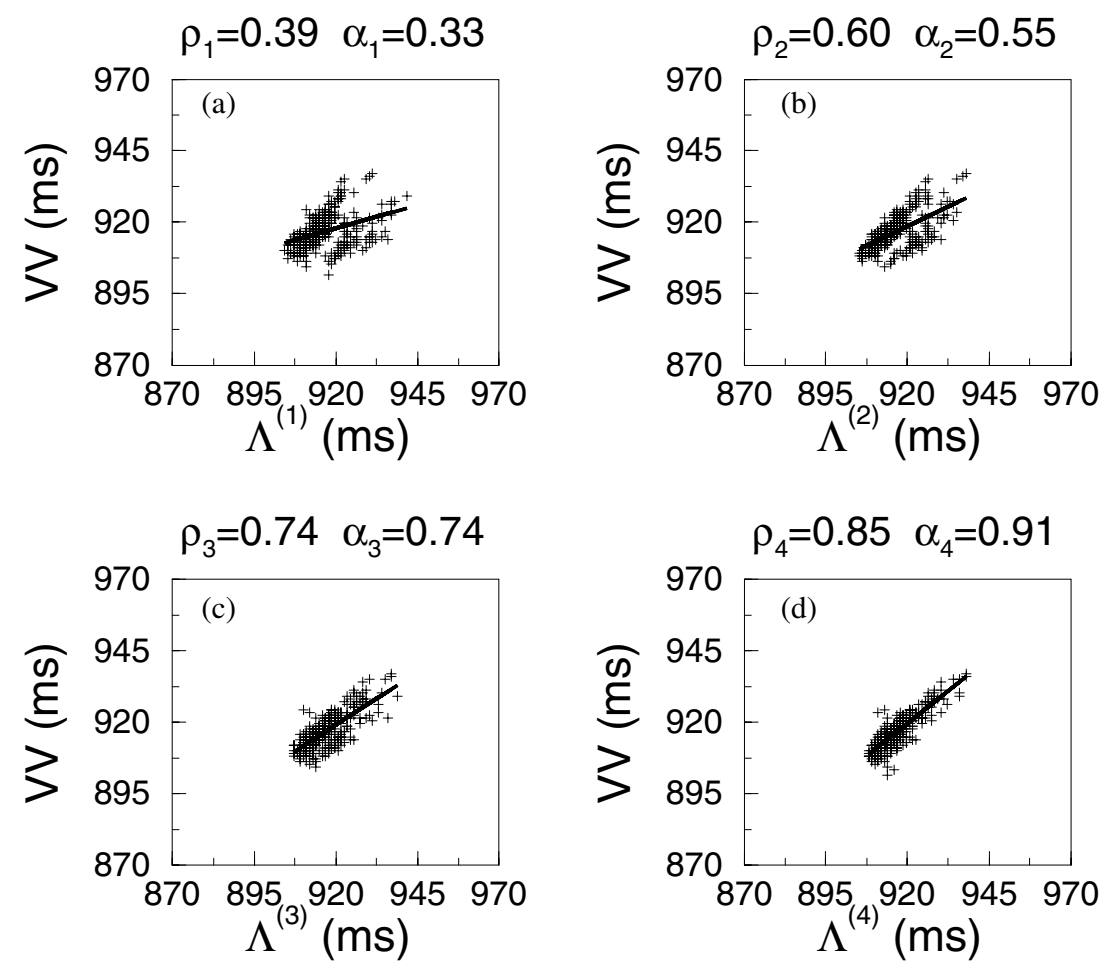

Figure 6. Atrial flutter. Interventricular intervals $V V_{i}$ are plotted as a function of the grouped interatrial intervals $\Lambda_{i}$. Graphs (a)-(d) correspond to interpretations in panels (a)(d) in Fig. 5. The graphs also show the linear regression lines of the data points with the correlation coefficients $\rho$ and regression coefficients $\alpha$ appearing in the figure titles. The linear regression lines are computed by using straightforward least-squares fitting.

time using interpretation $\mathrm{D}$ during the $4: 1$ block is greater than the conduction time during sinus rhythm.

Model results. Analysis of the atrial flutter data revealed that interpretation D (Fig. 5) is the most likely conduction pattern. Under that assumption a constant conduction time of approximately $164 \mathrm{~ms}$ is a good estimate of the real conduction properties in the atrial flutter data. The recovery curve equation (2) thus becomes very simple, since $f\left(R A_{k}\right)=A V_{\min }$, and the resulting parameter space of the model is then three-dimensional $\left(A V_{\min }, \theta, \Delta\right)$. By fixing $A V_{\min }=164 \mathrm{~ms}$, it is a simple task to scan the $(\theta, \Delta)$ parameter plane. An appropriate range for the parameter $\theta$ (the refractory period after a conducted beat) is $0 \mathrm{~ms} \leq \theta \leq 1000 \mathrm{~ms}$ in which the upper limit equals four maximum interatrial intervals $\left(A A_{\max } \approx\right.$ $250 \mathrm{~ms}$ ). A reasonable parameter range of $\Delta$ (the prolongation due to blocked atrial impulses) is $0 \mathrm{~ms} \leq \Delta \leq 230 \mathrm{~ms}$ in which the upper limit is determined by the average interatrial interval $\left(A A_{\text {mean }} \approx 230 \mathrm{~ms}\right)$. For each parameter combination on this parameter plane the results of the area measure $D_{T}$ and the Kolmogorov- 


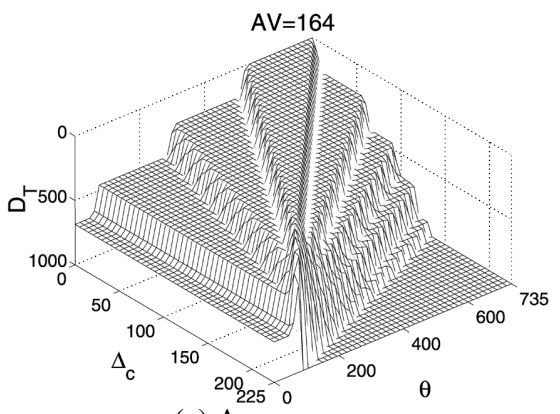

(a) Area measure.

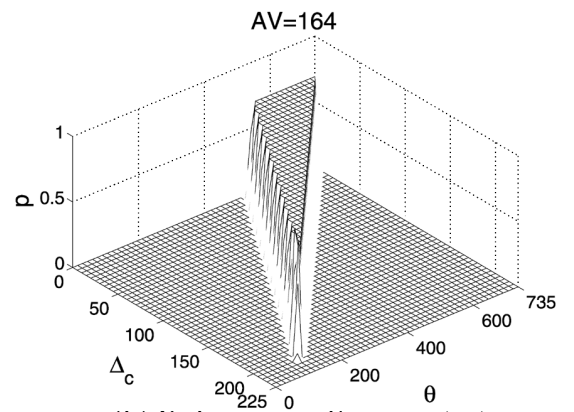

(b) Kolmogorov-Smirnov test.

Figure 7. Atrial flutter. Scanning the $(\Delta, \theta)$ parameter plane. Graphs (a) and (b) show the results of the area measure $D_{T}$ and the Kolmogorov-Smirnov test $p$, respectively.
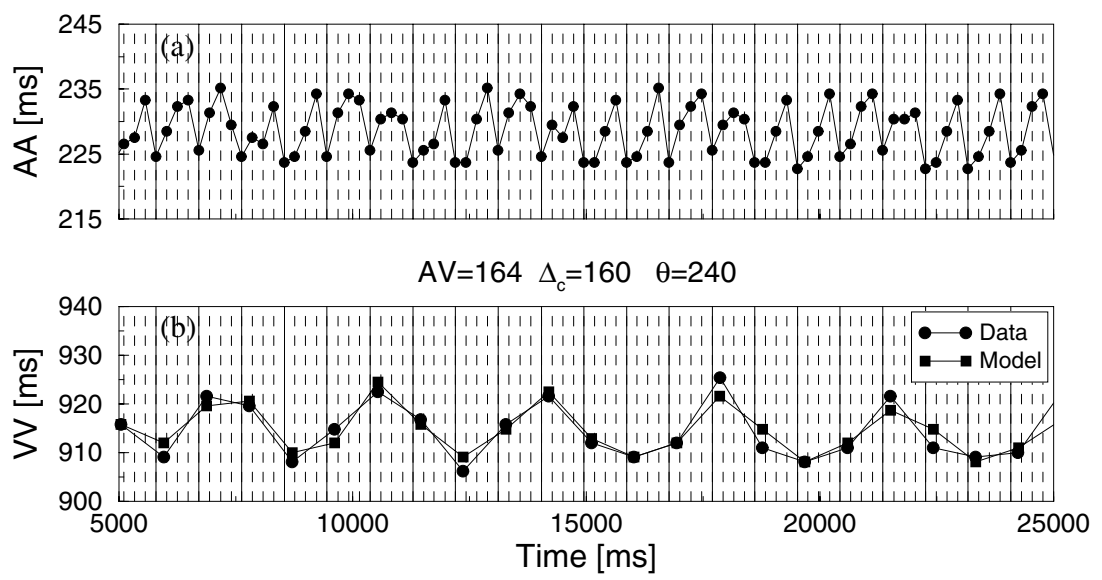

Figure 8. Atrial flutter. Interatrial and interventricular intervals of data and model. The upper panel shows the interatrial intervals obtained from the data set, and in the lower panel the interventricular intervals of model and data are shown. The vertical lines in both panels are the times of the atrial events (the solid lines being the conducted events and the dashed lines the blocked events).

Smirnov test $p$ are computed and plotted (Fig. 7). In both subfigures a triangleshaped region of parameter space emerges in which there is good agreement between model and data. The stepwise structure observed in Fig. 7(a) originates from the relatively equidistantly spaced atrial events throughout the recording time. The equidistant interatrial intervals effectively discretize time into units of the mean interatrial interval. A specific parameter combination, which yields good agreement between model and data, is chosen in order to look at the model output on a beat-to-beat basis. We choose the parameter combination: $\left(A V_{\min }, \Delta, \theta\right)=$ $(164,160,240)$ for which $D_{T}=1.81$ and $p=0.69$. There is generally very good agreement on a beat-to-beat basis between model and recording. Figure 8 shows for the aforementioned parameter combination an example of how well the model compares to the recording. 
Table 2. Statistics of atrial fibrillation data.

\begin{tabular}{lccc}
\hline Intervals & $A A$ & $V V^{\text {rec }}$ & $V V^{\text {model }}$ \\
\hline Number of intervals & 2055 & 447 & 445 \\
Minimum & $52 \mathrm{~ms}$ & $352 \mathrm{~ms}$ & $478 \mathrm{~ms}$ \\
Maximum & $241 \mathrm{~ms}$ & $1000 \mathrm{~ms}$ & $959 \mathrm{~ms}$ \\
Average & $146 \mathrm{~ms}$ & $669 \mathrm{~ms}$ & $671 \mathrm{~ms}$ \\
Standard deviation & $16 \mathrm{~ms}$ & $105 \mathrm{~ms}$ & $100 \mathrm{~ms}$ \\
Coefficient of variation & 0.11 & 0.16 & 0.15 \\
\hline
\end{tabular}

\section{Atrial fibrillation.}

Data analysis. Key statistical numbers of the atrial fibrillation data are summarized in Table 2. In atrial fibrillation the average interatrial interval is $A A_{\text {mean }}=$ $146 \pm 16 \mathrm{~ms}$, and the average interventricular interval is $V V_{\text {mean }}=669 \pm 105 \mathrm{~ms}$. The coefficient of variation $(\mathrm{CV}=$ standard deviation/mean) of the ventricular beats is 0.16 (Meijler et al., 1996). In atrial fibrillation the conduction ratio varies throughout the recording time, but on average the conduction ratio is 4.59. The ventricular rhythm relative to the atrial rhythm is thus slower in atrial fibrillation than in atrial flutter. The autocorrelation function of the atrial activation times shows linear correlation for small lags, whereas the autocorrelation function of the ventricular interbeat intervals shows no linear correlation (Fig. 3) (Goldstein and Barnett, 1967; Meijler et al., 1968; Bootsma et al., 1970; Stackee et al., 1971; Hashida et al., 1978; Cohen et al., 1983).

Model results. We cannot establish which atrial events are conducted in atrial fibrillation. Consequently, the model cannot be tailored to a certain interpretation of the data, for example a constant conduction time. Therefore, we use the recovery curve equation (2) with all three parameters $\left(A V_{\min }, \alpha, \tau\right)$ to describe the conductive properties of the atrial fibrillation data. The scanning process is intricate due to the five-dimensional parameter space and the fact that none of the parameters can be estimated precisely from the atrial fibrillation data. We have taken the following approach. In atrial fibrillation the interatrial and interventricular intervals are generally smaller than in atrial flutter. Therefore it follows that the refractory parameters $\theta$ and $\Delta$ are smaller in magnitude than is the case in atrial flutter. From Table 2 we can crudely deduce the ranges: $0 \mathrm{~ms} \leq \theta \leq V V_{\text {mean }} \approx 700 \mathrm{~ms}$ and $0 \mathrm{~ms}$ $\leq \Delta \leq A A_{\text {mean }} \approx 150 \mathrm{~ms}$. The parameters of the recovery curve $\left(A V_{\min }, \alpha, \tau\right)$ are scanned in the ranges: $0 \mathrm{~ms} \leq A V_{\min } \leq 150 \mathrm{~ms}, 0 \mathrm{~ms} \leq \alpha \leq 350 \mathrm{~ms}$, and $10 \mathrm{~ms}$ $\leq \tau \leq 150 \mathrm{~ms}$ using stepsizes of 10-25 ms depending on the range of the particular parameter. Only parameter combinations fulfilling predefined values of the area measure and Kolmogorov-Smirnov test are stored in the introductory scans. These parameter combinations are then sorted with respect to the best results of the area measure and the Kolmogorov-Smirnov test. The sorted parameters give 


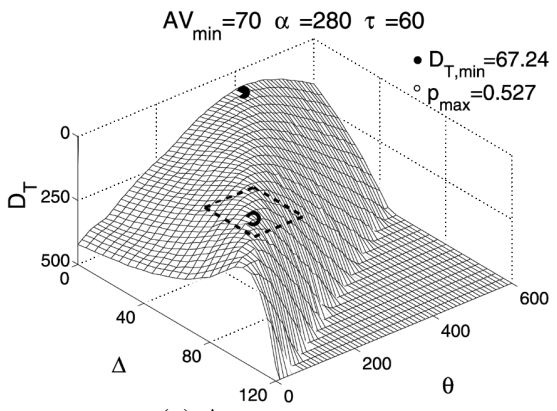

(a) Area measure.

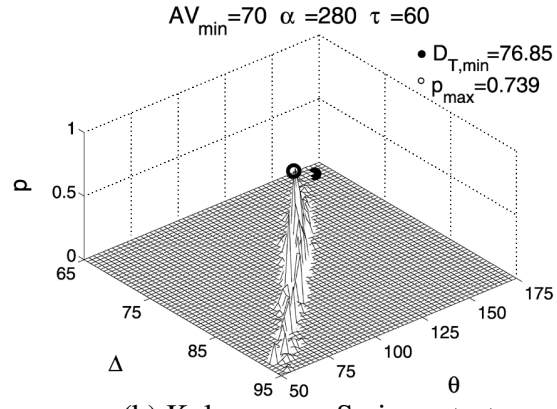

(b) Kolmogorov-Smirnov test

Figure 9. Atrial fibrillation. Scanning the $(\Delta, \theta)$ parameter plane. Graphs (a) and (b) show the results of the area measure $D_{T}$ and the Kolmogorov-Smirnov test $p$, respectively.

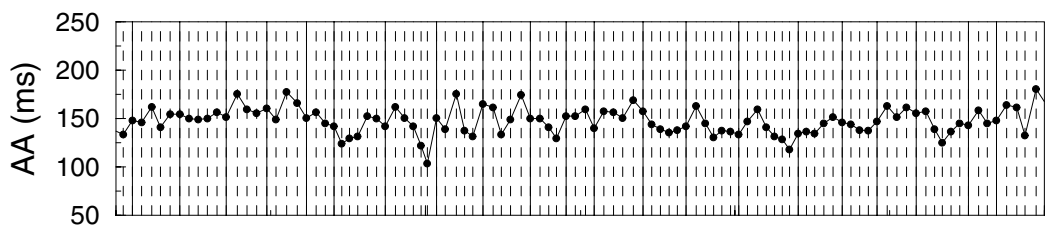

$\mathrm{AV}_{\min }=65 \quad \alpha=290 \quad \tau=55 \quad \Delta=81 \quad \theta=114$

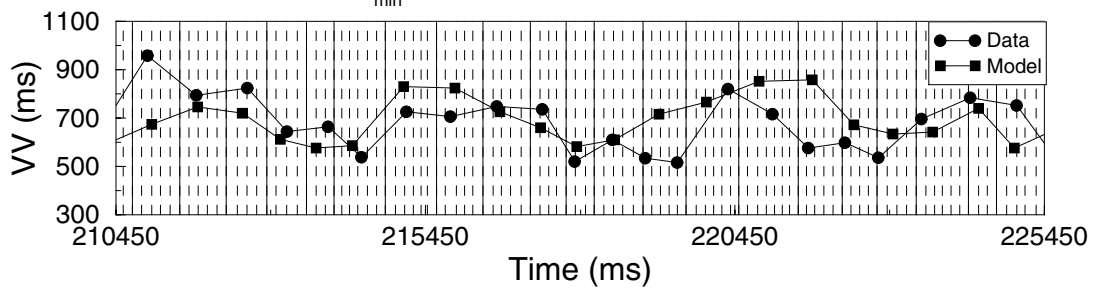

Figure 10. Atrial fibrillation. Interatrial and interventricular intervals of data and model. The upper panel shows the interatrial intervals obtained from the data set, and in the lower panel the interventricular intervals of model and data are shown. The vertical lines in both panels are the times of the atrial events (the solid lines being the conducted events and the dashed lines the blocked events).

a good idea of regions in the parameter space where good fits are found, and the parameter ranges for the next scan can thus be narrowed substantially. Finally, after a number of scans of still smaller regions, a certain recovery curve can be chosen, and the $(\theta, \Delta)$ parameter plane is scanned while keeping this recovery curve fixed. Figure 9 shows the result of scanning the $(\theta, \Delta)$ parameter plane for the recovery curve $\left(A V_{\min }, \alpha, \tau\right)=(70,280,60) \mathrm{ms}$. Figure 9 shows that reasonable agreement between model and data can be found for certain parameter combinations with respect to the area measure and the Kolmogorov-Smirnov test. It is observed that the high $p$-values are located on the ridge formed by the results of the area measure (Fig. 9).

An example of the model output stemming from a point in parameter space with good model agreement is chosen to show the beat-to-beat output (Fig. 10). 

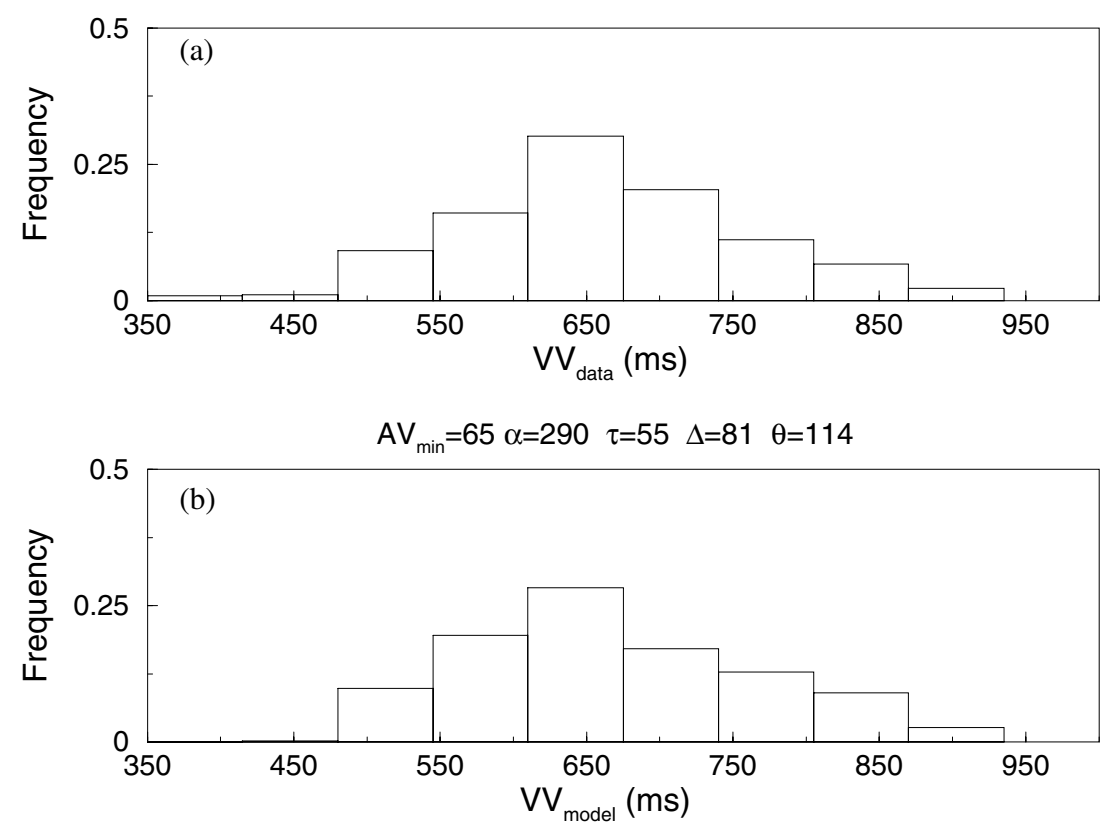

Figure 11. Panels (a) and (b) show the normalized distributions of recorded and predicted interventricular intervals, respectively.

The ventricular beat-to-beat variation shows that the model does not completely capture the ventricular response on a beat-to-beat basis. For the parameter combination used in Fig. 10 the Kolmogorov-Smirnov test yields $p=0.96$, the area measure is $D_{T}=90.0$, and finally the predicted conduction ratio is $C R=4.59$, which is the same as for the recorded data. The normalized histograms of the recorded and predicted interventricular intervals look very similar (Fig. 11). The statistics of the predicted ventricular response are found in Table 2.

\section{Discussion}

This work has presented a new technique to determine parameters in a mathematical model of the AV node based on observed atrial and ventricular activity. Given the measured atrial activation times, the times of ventricular activation are computed. The computed activation times are compared with the observed activation times as the parameters describing AV node function are varied. Parameters which give the best statistical correspondence between the computed and observed activation times are identified by scanning the parameter space of the model.

Comparison with previous models of AV nodal function. Our approach differs in important ways from earlier studies that developed theoretical models of the AV node. Some earlier studies developed theoretical models of the AV node based 
on independent tests of $\mathrm{AV}$ nodal function, for example, by testing $\mathrm{AV}$ nodal conduction during a variety of stimulation protocols in which atrial inputs are systematically changed (Heethaar et al., 1973a,b; Shrier et al., 1987; Talajic et al., 1991). Since in the setting of atrial fibrillation such stimulation protocols are not feasible, it is necessary to consider statistical features of the dynamics in order to set the parameters. A similar approach was considered earlier (Cohen et al., 1983; Zeng and Glass, 1996) which showed how unimodal or bimodal histograms of RR intervals could arise from modifications of parameters in AV nodal models. However, those earlier papers did not develop systematic methods for setting parameters based on comparisons between observed and computed histograms.

In atrial flutter and atrial fibrillation there are large numbers of nonconducted beats, and therefore the current model puts emphasis on the influence of these concealed beats on $\mathrm{AV}$ nodal function. At the moment, there is no general agreement of how to include the effects of concealed beats. The current work assumes that each concealed beat leads to a prolongation of the refractory period by a constant amount that does not depend on the timing of the concealed beat. Although there is evidence that the effects of concealed beats do depend on their timing (van Capelle et al., 1971; Heethaar et al., 1973b), there is no consensus on how to develop a quantitative model for this function.

One of the interesting properties of the current model is that during the rapid stimulation rates that arise in atrial flutter and atrial fibrillation, the number of conducted beats would be inversely related to the frequency of the atrial inputs provided the properties of the AV node remain fixed. In the current model, this effect arises since each blocked beat leads to an increase in the refractory time of the AV node. This is in accord with experiments in isolated rabbit hearts, in which ventricular rate decreased as the average stimulation rate was increased during irregular atrial pacing (Chorro et al., 1990). This behavior is not shared by all theoretical models of the AV node. For example, in the model developed by Cohen et al. (1983), each atrial input contributes to the depolarization of the AV node so that increased atrial stimulation would lead to an increased ventricular response.

A paradoxical decrease in ventricular response with increased irregular atrial activity was also observed in a set of coupled nonlinear differential equations that were proposed by Meijler et al. (1996) as a model of the AV node. The current work is complementary to this earlier work. In the earlier work, a large number of parameters describe the ionic channels of the model cells. The sensitivity of the model to changes in these parameters is not known, and systematic searches of the high dimensional parameter space associated with such models would be difficult if not impossible to carry out.

Limitations of the model. There are several time-dependent changes of the AV node during rapid stimulation. The conduction time through the AV node does not depend solely on recovery time but also on the past history, as described by the concepts of fatigue and facilitation (Heethaar et al., 1973a; Billette and Nattel, 1994). 
Incorporation of fatigue and facilitation into mathematical models of the AV node can lead to a variety of subtle dynamical effects including changes in ratio of atrial to ventricular beats during stimulation at fixed frequency (Talajic et al., 1991), or alternation of the conduction time through the AV node during atrial stimulation at a fixed delay after ventricular activation (Sun et al., 1995; Amellal et al., 1996). Though inclusion of fatigue and facilitation would have been possible in the current model, the incorporation of extra parameters would have made it much more difficult to scan the parameter space. Furthermore, fatigue and facilitation are especially important during rapid activation in which each atrial stimulus is conducted to the ventricles or when a small fraction of atrial activations are blocked in the AV node (Talajic et al., 1991; Sun et al., 1995; Amellal et al., 1996).

Another factor omitted in the current work is the feedback from ventricular contraction to AV nodal and atrial activity. This feedback affects AV nodal conductive and refractory properties (Damato et al., 1972; Pick and Langendorf, 1979; Meijler et al., 1996) and can also affect the timing of atrial impulses during atrial flutter (Lammers et al., 1991; Ravelli et al., 1994; Stambler and Ellenbogen, 1996).

Clearly, an ionic model of the heart along the lines proposed by Meijler et al. (1996) should be able to reproduce all important functional properties of the AV node if subjected to a wide range of inputs. However, such models are extremely complex. We believe that analysis of simplified models, such as that proposed here, offers a means to identify key functional properties of the AV node underlying the observed complex dynamics. Thus, the current approach is complementary to the development of detailed ionic models.

\section{CONClusions}

This work analyzes and models the ventricular response during atrial flutter and atrial fibrillation. We hypothesize that concealed beats lead to an increase in the refractory period of the AV node. Using a highly simplified model, parameters in the model are determined by comparing statistical properties of the measured ventricular activity with the computed response given the atrial input. This methodology should be able to help elucidate the determinants of the ventricular response to atrial fibrillation in patients in whom simultaneous recordings of atrial and ventricular activity are available. In particular, these techniques should help to resolve the relative roles of atrial activity and $\mathrm{AV}$ nodal function in a single patient as the ventricular response changes due to drugs or circulating hormones during the course of the day. Control of the ventricular response to atrial fibrillation is one of the two principal strategies in atrial fibrillation therapy (the other being sinus rhythm maintenance). By providing a way to analyze the determinants of the ventricular response to atrial fibrillation, our technique may be useful for the evaluation and comparision of different approaches to control the ventricluar response. 


\section{ACKNOWLEDGEMENTS}

This research has been partially supported by the MITACS National Center of Excellence, the Canadian Institute for Health Research, the Research Resource for Complex Physiologic Signals (NIH), and the Humboldt Foundation.

\section{REFERENCES}

Amellal, F., K. Hall, L. Glass and J. Billette (1996). Alternation of atrioventricular nodal conduction time during atrioventricular reentrant tachycardia: are dual pathways necessary? J. Cardiovasc. Electrophysiol. 7, 943-951.

Billette, J. and S. Nattel (1994). Dynamic behavior of the atrioventricular node: a functional model of interaction between recovery, facilitation, and fatigue. J. Cardiovasc. Electrophysiol. 5, 90-102.

Bootsma, B. K., A. T. Hoelen, J. Strackee and F. L. Meijler (1970). Analysis of R-R intervals in patients with atrial fibrillation at rest and during exercise. Circulation 41, 783-794.

Chorro, F. J., C. J. H. J. Kirchof, J. Brugada et al. (1990). Ventricular response during irregular atrial pacing and atrial fibrillation. Am. J. Physiol. 259, H1015-H1021.

Cohen, R. J., R. D. Berger and T. E. Dushane (1983). A quantitative model for the ventricular response during atrial fibrillation. IEEE Trans. Biomed. Eng. 30, 769-781.

Damato, A. N., O. J. Varghese, S. H. Lau, J. J. Gallagher and G. A. Bobb (1972). Manifest and concealed reentry. A mechanism of AV nodal Wenckebach phenomenon. Circ. Res. 30, 283-292.

Decherd, G. M. and A. Ruskin (1946). The mechanism of the Wenckebach type of A-V block. Br. Heart J. 8, 6-16.

Goldstein, R. E. and G. O. Barnett (1967). A statistical study of the ventricular irregularity of atrial fibrillation. Comput. Biomed. Res. 1, 146.

Hashida, E., N. Yoshitani and T. Tasaki (1978). A study on the irregularity of the sequence of R-R intervals in chronic atrial fibrillation in man based on the time series analysis and the information theory. Jpn. Heart J. 19, 839-851.

Heethaar, R. M., J. J. D. van Der Gon and F. L. Meijler (1973a). Mathematical model of AV conduction in the rat heart. Cardiovasc. Res. 7, 105-114.

Heethaar, R. M., R. M. De Vos Burchart, J. J. D. van Der Gon and F. L. Meijler (1973b). A mathematical model of AV conduction in the rat heart. II. Quantification of concealed conduction. Cardiovasc. Res. 7, 542-556.

Lammers, W. J. E. P., F. Ravelli, M. Disertori, R. Antolini, F. Furlanello and M. A. Allessie (1991). Variations in human atrial flutter cycle length induced by ventricular beats: evidence of a reentrant circuit with a partially excitable gap. J. Cardiovasc. Electrophysiol. 2, 375-387.

Langendorf, R. (1948). Concealed A-V conduction: the effect of blocked impulses on the formation and conduction of subsequent impulses. Am. Heart J. 35, 542-552. 
Lewis, T. and A. M. Master (1925). Observations upon conduction in the mammalian heart. A-V conduction. Heart 12, 209-269.

Meijler, F. L., J. Jalife, J. Beaumont and D. Vaidya (1996). AV nodal function during atrial fibrillation: the role of electrotonic modulation of propagation. J. Cardiovasc. Electrophysiol. 7, 843-861.

Meijler, F. L., J. Stackee, F. J. L. van Capelle and J. du Perron (1968). Computer analysis of the RR interval-contractility relationship during random stimulation of the isolated heart. Circ. Res. 22, 695-702.

Moe, G. K. and J. A. Abildskov (1964). Observations on the ventricular dysrhythmia associated with atrial fibrillation in the dog heart. Circ. Res. 14, 447-460.

Pavri, B. B., D. Kocovic and M. Hanna (1999). Long-short RR intervals and the right bundle branch. J. Cardiovasc. Electrophysiol. 10, 121-123.

Pick, A. and R. Langendorf (1979). Interpretation of Complex Arrhythmias, Philadelphia: Lea \& Febiger.

Press, W. H., S. T. Teukolsky, W. T. Vetterling and B. P. Flannery (1993). Numerical Recipes in C: The Art of Scientific Computing, Cambridge: Cambridge University Press. Also see: http://www.ulib.org/webRoot/Books/Numerical_Recipes/ bookcpdf.html.

Ravelli, F., M. Disertori, F. Gozzi, R. Antolini and M. A. Allessie (1994). Characterization of atrial flutter. Studies in man after open heart surgery using fixed atrial electrodes. Circulation 89, 2107-2116.

Shrier, A., H. Dubarsky, M. Rosengarten, M. R. Guevara, S. Nattel and L. Glass (1987). Prediction of complex atrioventricular conduction rhythms in humans with use of the recovery curve. Circulation 76, 1196-1205.

Stackee, J., A. J. Hoelen, N. E. Zimmerman and F. L. Meijler (1971). Artificial atrial fibrillation in the dog. An artifact? Circ. Res. 28, 441-445.

Stambler, B. S. and K. A. Ellenbogen (1996). Elucidating the mechanisms of atrial flutter cycle length variability using power spectral analysis techniques. Circulation 94, 2515-2525.

Sun, J., F. Amellal, L. Glass and J. Billette (1995). Alternans and period-doubling bifurcations in atrioventricular nodal conduction. J. Theor. Biol. 173, 79-91.

Talajic M., D. Papadatos, C. Villemaire, L. Glass and S. Nattel (1991). A unified model of atrioventricular nodal conduction predicts dynamic changes in Wenckebach periodicity. Circ. Res. 68, 1280-1293.

Task Force of the European Society of Cardiology and the North American Society of Pacing and Electrophysiology: heart rate variability (1993). Standards of measurement, physiological interpretation, and clinical use. Circulation 93, 1043-1065.

van Capelle, F. J. L., J. C. du Perron and D. Durrer (1971). Atrioventricular conduction in isolated rat heart. Am. J. Physiol. 221, 284-290.

Waxman, M. B., L. Yao, D. A. Cameron and J. A. Kirsh (1991). Effects of posture, valsalva maneuver and respiration on atrial flutter rate: an effect mediated through cardiac volume. J. Am. Coll. Cardiol. 17, 1545-1552. 
Wells, J. L., W. A. H. MacLean, T. N. James and A. L. Waldo (1979). Characterization of atrial flutter. Studies in man after open heart surgery using fixed atrial electrodes. Circulation 60, 665-673.

Zeng, W. and L. Glass (1996). Statistical properties of heartbeat intervals during atrial fibrillation. Phys. Rev. E 54, 1779-1784.

Received 13 February 2002 and accepted 1 August 2002 